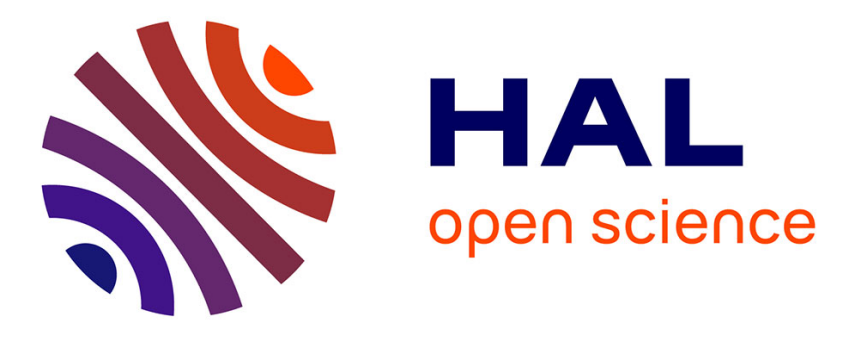

\title{
Experimental multiscale measurements for the mechanical identification of a cortical bone by digital image correlation
}

Manh-Tu Nguyen, Jean-Marc Allain, Hakim Gharbi, Christophe Desceliers, Christian Soize

\section{To cite this version:}

Manh-Tu Nguyen, Jean-Marc Allain, Hakim Gharbi, Christophe Desceliers, Christian Soize. Experimental multiscale measurements for the mechanical identification of a cortical bone by digital image correlation. Journal of the mechanical behavior of biomedical materials, 2016, 63, pp.125-133. 10.1016/j.jmbbm.2016.06.011 . hal-01337358

\section{HAL Id: hal-01337358 https://hal.science/hal-01337358}

Submitted on 25 Jun 2016

HAL is a multi-disciplinary open access archive for the deposit and dissemination of scientific research documents, whether they are published or not. The documents may come from teaching and research institutions in France or abroad, or from public or private research centers.
L'archive ouverte pluridisciplinaire HAL, est destinée au dépôt et à la diffusion de documents scientifiques de niveau recherche, publiés ou non, émanant des établissements d'enseignement et de recherche français ou étrangers, des laboratoires publics ou privés. 


\title{
Experimental multiscale measurements for the mechanical identification of a cortical bone by digital image correlation
}

\author{
Manh-Tu Nguyen ${ }^{\mathrm{a}}$, Jean-Marc Allain ${ }^{\mathrm{b}}$, Hakim Gharbi ${ }^{\mathrm{b}}$, Christophe Desceliers ${ }^{\mathrm{a}}$, Christian Soize ${ }^{\mathrm{a}, *}$ \\ ${ }^{a}$ Université Paris-Est, Laboratoire Modélisation et Simulation Multi-Echelle, MSME UMR 8208 CNRS, 5 bd Descartes, 77454 Marne-La-Vallée, Cedex 2, France. \\ ${ }^{b}$ Ecole Polytechnique, Laboratoire de Mécanique des Solides, 91128, Palaiseau cedex, France.
}

\begin{abstract}
The implementation of the experimental methodology by optical measurements of mechanical fields, the development of a test bench, the specimen preparation, the experimental measurements, and the digital image correlation (DIC) method, have already been the object of research in the context of biological materials. Nevertheless, in the framework of the experimental identification of a mesoscopic stochastic model of the random apparent elasticity field, measurements of one specimen is required at both the macroscopic scale and the mesoscopic scale under one single loading. The nature of the cortical bone induces some difficulties, as no single speckled pattern technique is available for simultaneously obtaining the displacement at the macroscopic scale and at the mesoscopic scale. In this paper, we present a multiscale experimental methodology based on (i) an experimental protocol for one specimen of a cortical bone, (ii) its measuring bench, (iii) optical field measurements by DIC method, (iv) the experimental results, and (v) the multiscale experimental identification by solving a statistical inverse problem.
\end{abstract}

Keywords: Multiscale measurements, Multiscale experiments, Optical imaging, DIC method, Digital image correlation method, Multiscale identification, Heterogeneous microstructure, Random elasticity field, Mesoscale, Statistical inverse problem

\section{Preambule}

This paper is related to a work Nguyen (2013) devoted to the multiscale identification of the elasticity field at mesoscale of a cortical bone by solving a statistical inverse problem. Such a work had required to develop two different and complementary parts. The first one was relative to the development of a theoretical formulation for the statistical inverse problem that had the capability to identify the elasticity random field of the heterogeneous microstructure by using a multiscale digital image correlation technique performed simultaneously at macroscale and at mesoscale. This first part of the work was already published in Nguyen et al. (2015). The second part was related to the experimental part devoted to the cortical bone. This experimental part has been extremely difficult to carry out in order to obtain reliable experimental outcomes. This is the reason why the authors publish this paper for explaining the experimental procedure that has been followed for the experimental protocol, for the preparation of the cortical bone specimen, for the test bench, and for the test procedure. This part has never been published. Nevertheless, there are a few overlaps concerning (i) the experimental results that have already been presented in Nguyen et al. (2015) in order to illustrate the theory proposed for the multiscale identification and (ii) the theory for the multiscale statistical inverse problem that is detailed in Nguyen et al. (2015), and which is briefly summarized at the end of this paper in order to give a self-contained overview to the reader.

\footnotetext{
${ }^{*}$ Corresponding author

Email address: christian.soize@univ-paris-est.fr (Christian Soize )
}

\section{Introduction}

The experimental identification of microstructural morphology by image analysis began in the 1980s Jeulin (1987, 1989, 2001), and has led to significant advances for the identification of the elastic properties of materials at the macroscale and/or mesoscale. Many works could be cited among which Besnard et al. (2006); Bornert et al. (2009); Hild (2002); Hild et al. (1999); Hild \& Roux (2006, 2012); Kahnietter et al. (1994); Rethore et al. (2008); Roux \& Hild (2008); Roux et al. (2002, 2008); Vendroux \& Knauss (1998a b) for field measurements using digital image correlation (DIC) (see for instance Pan et al. (2009, 2006); Schreier et al. (2000); Sutton et al. (2008)), including software developments, Bornert et al. (2010); Hild et al. (2002) for multiscale field measurements, Bonnet \& Constantinescu (2005); Constantinescu (1995); Geymonat et al. (2002); Geymonat \& Pagano (2003) for inverse problems in elasticity, Avril et al. (2008a); Avril \& Pierron (2007); Avril et al. (2008b); Calloch et al. (2002); Chevalier et al. (2001); Madi et al. (2007) for identification methods from full-field measurements in linear and nonlinear elasticity, and Baxter \& Graham (2000); Graham et al. (2003) for stochastic aspect of random media using a moving-window technique.

In this paper, the heterogeneous material is made up of a cortical bone for which the microstructure is very complex, random and presents a hierarchical structure. At the macroscopic scale, such a material is generally assumed to be deterministic and homogeneous. Consequently, its elasticity tensor can be identified using experimental measurements of the elastic 
displacement field at the macroscopic scale. Furthermore, at the microscopic level, such a material is heterogeneous and random, and cannot be described in terms of its constituents. Therefore, a mesoscopic scale is introduced between the macroscopic scale and the microscopic scale, for which the apparent elasticity field of the microstructure is modeled by a random field. The identification procedure of the prior stochastic model of this apparent elasticity field is detailed in Nguyen (2013); Nguyen et al. (2015) for which the measurements of the displacement field are required both at the macroscopic scale and at the mesoscopic scale.

The implementation of the experimental methodology by optical measurements of mechanical fields, the development of a measuring bench, the specimen preparation, the experimental measurements, and the DIC method, have already been the object of research in the context of biological materials Bertolino et al. (2007); Bornert et al. (2010); Chauvet et al. (2010); Gusachenko et al. (2012); Heripre et al. (2007); Houssen et al. (2011); Kalouche et al. (2010); Yang et al. (2012, 2010); Yamaguchi et al. (2011).

In this paper, new advances are proposed to circumvent the difficulties induced by the complex nature of the cortical bone and the need to observe for the same loading two very different scales. A suitable protocol is required to apply the external loads to the specimen, and to obtain images by optical measurements of the displacement field in the lightened face with a sufficient contrast at both the mesoscopic scale and the macroscopic scale. Furthermore, the measurements of the elastic displacement field at mesoscopic and macroscopic scales have to be performed for a single loading applied to the specimen.

We present (i) an experimental methodology based on optical field measurements by the DIC method, (ii) an experimental protocol for one specimen of cortical bone, (iii) its measuring bench, and (iv) the experimental results.

\section{Methodology for the elastic displacement-field measure- ments by using the digital image correlation method}

The field measurements by digital image correlation method was introduced and has been developed in many works since the early 1990's (see the references given in Section 1). For a given spatial resolution, the elastic displacement field of the lightened face of the specimen, submitted to external loads, can be obtained by comparing two images. The first image corresponds to the undeformed specimen (reference image) while the second one corresponds to the deformed specimen under the external load (deformed image). The comparison of these two images relies on the digital image correlation under the assumption that the contrast is locally preserved in the vicinity of each point where the displacement is measured.

There exists a large number of approaches for obtaining the images. It depends on the nature of the contrast, the correlation coefficient that is estimated using the digitalized images, and which requires an optimization algorithm. Most of the implementations of the image correlation method rely on the use of an optical CCD camera, equipped with an optical lens adapted to the scale of the observed structure. At the macroscopic scale, the displacement field (that has to be measured) varies between a few millimeters and a few centimeters, or more. Such an imaging method is usually free of any technical problem related to the lighting, the alignment or the field depth. Images are then recorded by a computer equipped with a video card (for which the frequency has been adapted to the measured specimen), a data acquisition system, and a devoted processing software. The images are analyzed by the image correlation method in grey levels. For each pixel, the signal is represented by a scalar corresponding to a grey level. The colored images have not been used because the color induces two major difficulties. The first one is related to the definition of the gap between two colors that are coded by a scalar indicator; consequently, the definition of a correlation coefficient based on a distance between two images remains an open problem for images in color. The second one is related to the implementation of a usual color camera, which uses a color filter located in front of its CCD sensor, implying that some pixels are sensitive to blue, some others are sensitive to red, and others are sensitive to green. In consequence, the signal is undersampled with a factor four, which is detrimental to the performance of the image correlation method.

It is also possible to obtain much smaller images with a macrophotographic lens or with an optical microscope. The dimensions of the observed area can then be less than one square millimeter or even a tenth of a square millimeter. If the magnification is increased, some difficulties appear due to the low amount of available light, which requires strong enough lighting. Another problem concerns the field depth, which decreases as the magnification grows. In order to obtain sharp images throughout the observation area, it is then necessary to ensure the flatness of the observed lightened face, which requests an adequate polishing. However, cortical bone is delicate to polish as it is very sensitive to chemical agents, temperature and is difficult to handle. In addition, the alignment of the optical axis with the normal to this face should be set carefully. Another difficulty is the control of the working distance between the observation system and the observed specimen. Indeed, a variation in this distance will blur the image. Small variations will lead to an artificial magnification, which generates unphysical strains (associated to a dilatation). Large variations lead to non-usable images. The focus distance is fixed and the system focusses automatically by moving the full optical setup. In the framework of the classical optics, the resolution limit of optical cameras is related to the wavelength of the light used. In practice, for a standard white sub-lighting, and for a high quality optical system, the lower limit of a pixel is approximatively $0.5 \times 10^{-6} \mathrm{~m}$. It is possible to have pixels associated to smaller lengths, leading to oversampling, but the adjacent pixels are then correlated. A higher magnification can be reached with other imaging systems as the scanning electron microscopy (SEM) whose resolution can be $10^{-9} \mathrm{~m}$ with a field-emission scanning electron microscope. 


\section{Experimental protocol for the cortical bone and measur- ing bench}

In this section, the method for preparing a cortical bone specimen, measuring bench, the test procedure and the difficulties encountered are presented.
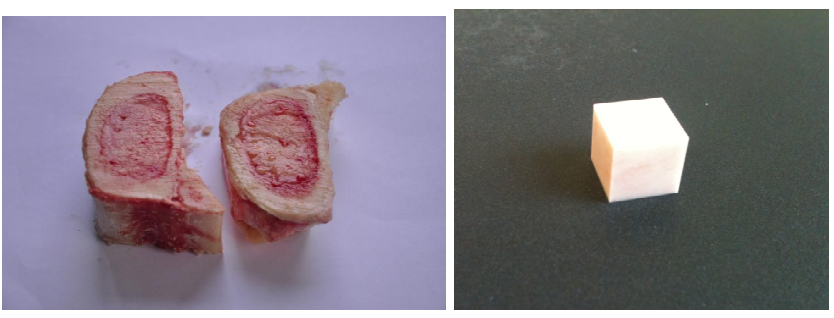

Figure 1: Beef cortical bone. Specimen before preparation (left) and after preparation (right) $\left(\right.$ cube with dimensions $0.01 \times 0.01 \times 0.01 \mathrm{~m}^{3}$ )

\subsection{Specimen preparation}

Biological tissues are among the most difficult material to be tested and to be modeled because of the high level of complexity of their structure the microscopic scale. It is the case for the cortical bone for which several scales have to be defined from $10^{-9} \mathrm{~m}$ to $10^{-3} \mathrm{~m}$. The cortical bone is a porous heterogenous anisotropic material that is very stiff and very resistant and for which, at the microscale, osteons, lamellae and canals can be identified. Cortical bone specimens coming from beef femurs have been used. For the cortical-bone specimen the macroscopic scale is $10^{-2} \mathrm{~m}$, the mesoscopic scale is $10^{-3} \mathrm{~m}$, and the scale of the microstructural heterogeneities is between $10^{-5} \mathrm{~m}$ and $10^{-6} \mathrm{~m}$.

The sample is extracted from a beef femur. The femur is initially cut into rough sections (see Fig. 1). Then the marrow, the nerves, and the cartilage attached to the bone have been removed. In a second step, the cleaned bone is cut with a diamond wire saw. The final shape of the specimen is a cube with dimension $0.01 \times 0.01 \times 0.01 \mathrm{~m}^{3}$. The maximum error on the dimension of its edges is 5\% (see Fig. 1). During all the procedure, we kept track of the orientation of the sample with respect to the initial bone, so that one face is parallel to the long axis of the bone (associated with the direction of the osteons). The third step consists in polishing the surfaces of the specimen with a disk polisher in order to ensure a sufficient flatness. During the polishing, the specimen is fixed at a specimen carrier by using a double sided adhesive tape. Then, parallel faces are obtained by pressing softly the specimen against the disk polisher. The observed face of the specimen is perpendicular to the long axis of the bone, so that we see the osteon.

In the last step, the observed face is covered with a speckled pattern. It is important to obtain a high contrast for the digital image correlation method. In the framework of the multiscale experiments devoted to the proposed identification procedure Nguyen (2013); Nguyen et al. (2015), it should be noted that a single speckled pattern is used for the optical measurements of the displacement fields both at the macroscopic and mesoscopic scales for the specimen submitted to a single external load. Three speckled pattern techniques have been tested for this biological material, among which two of them (the two first techniques presented hereinafter) failed to give a sufficient contrast. Experimental measurements presented in Section 4 have been obtained with the third speckled pattern technique. The first technique has consisted in spraying a black paint that yields good results at macroscopic scale but the paint drops were too big and the speckled pattern was not usable for the mesoscopic scale.

A speckled pattern of microbeads (Polybead microspheres, di-

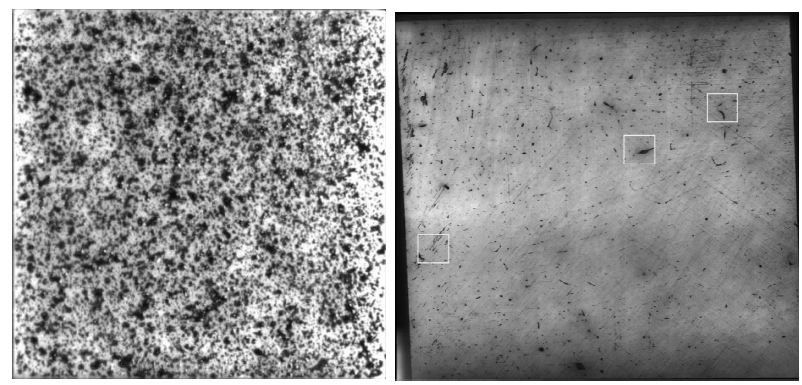

Figure 2: Observed face of the specimen, at the macroscopic scale: speckled pattern with black paint (left figure) and scratches (right figure)

ameter $4.5 \times 10^{-6} \mathrm{~m}$ ) was tested as a second technique. With this technique, the microbeads were placed in a colored ink solution and then deposited through a pipette on the observed face. After drying of the ink solution, the specimen was ready for performing the measurements but, the obtained pattern was too much heterogeneous, and it did not yield sufficient contrast for optical imaging (despite that good results could be obtained with SEM). For the last technique, a polishing paper with grit\#800 has been used to scratch the observed face in two perpendicular directions. Then, after coating the observed face with black ink, it is wiped such that the black ink mostly fills the pores and the scratches. Fig. 22 displays the resulting speckled patterns for the black paint (left) and for the scratches (right).

\subsection{Test bench}

The test bench is shown in Fig. 3.

The prepared specimen is placed inside a home-made vertical

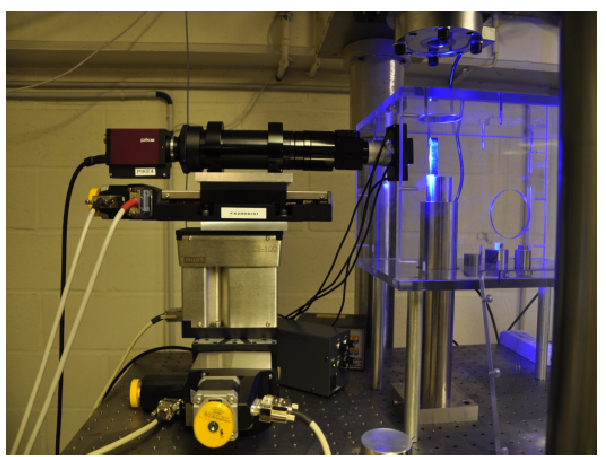

Figure 3: Photography of the optical setup in front of the compression machine.

uniaxial compression machine. The displacement of the top 
head of the machine is controlled, the bottom one being fixed. The force is recorded through a $10,000 \mathrm{~N}$ load cell. The sample is placed between two metallic cylinders inside the heads of the machine. The boundary conditions are thus not obvious to define. For the model, the following assumptions are used. The normal displacements of the face that is pressing against the lower fixed side of the UCM are locked while the corresponding tangential displacements are free. It is assumed that a constant surface-force field is applied to the opposite face that is pressing against the actuator of the machine. The tangential displacements are assumed to be free. The four other vertical faces of the specimen are free.

The sample is loaded at constant speed $\left(10^{-6} \mathrm{~m} / \mathrm{s}\right)$ up to 9,000 $\mathrm{N}$ : preliminary experiments have shown that this force allows for staying in the linear elasticity domain. A lighting source is placed inside the test bench, pointing out in the direction of the observed face. The images acquisition is performed by a single CCD camera (Pike F505, Allied Vision Technology, Germany) equipped with a x5 microscope lens (Mitutoyo, Japan), located in front the specimen. The pixel size is $\left(0.69 \times 10^{-6}\right) \times\left(0.69 \times 10^{-6}\right) \mathrm{m}^{2}$ with $2,452 \times 2,054$ pixels on the CCD sensor. This leads to an oversampled image, favorable for the DIC as soon as the correlation domain is still larger than the optical resolution.

The same setup is used for the acquisition of the images at microscale and at macroscale. The full surface is imaged by moving along the horizontal and the vertical directions. This can be done since the camera and its objective are installed on three motorized stages (horizontal movements: LS-110; vertical movements: ES-100, Micos, Germany): two motors are used to scan the surface, while the last one is use for the automatic focus. Nine images are taken along the horizontal direction, and ten images along the vertical one, insuring an overlap of at least $34 \%$. Each one of these 90 images defines a mesoscopic subdomain. The macroscopic image is built by combining the 90 mesoscopic images, using ImageJ stitching plugin Rasband (2013).

\subsection{Test procedure}

The test procedure is described as follows.

1. First, the maximal compression force that can be applied to the specimen is estimated in order to stay in the linear elastic domain. For that, several loading and unloading cases are applied to 4 dedicated specimens. As a result, the value $9,000 \mathrm{~N}$ is chosen.

2. Each one of the 90 images is acquired with $2,452 \times 2,054$ pixels for the specimen at rest (no applied load).

3. At macroscopic scale, a reference image is built in combining these 90 images (mosaic image) by using the software ImageJ. In addition, the image number 42 (located at the middle of the lightened face) is taken as the reference image for the mesoscopic scale.
4. The specimen is loaded at a speed of $10^{-6} \mathrm{~m} / \mathrm{s}$ up to the final value $9,000 \mathrm{~N}$. The displacement is then fixed and the acquisition of the 90 images is performed for the deformed specimen (deformed images). It should be noted that a very small relaxation of about $7 \%$ of the force has been observed during the test.

5. At macroscopic scale, the deformed image is built in combining these 90 deformed images (mosaic image). As previously, the image number 42 is taken as the deformed image for the mesoscopic scale.

6. At the macroscopic scale and at the mesoscopic scale, the displacement fields are calculated by the digital image correlation method. A spatial resolution of $10 \times 10$ - points grid is used for the macroscopic scale, which corresponds to $10 \times 10$ grid on the image obtained by stitching together the 90 images, while a $100 \times 100$ - points grid is used for the mesoscopic scale. The analysis has been carried out using the Correl Manu software. Taking into account the dimension of the specimen, the spatial resolution is $10^{-5} \mathrm{~m}$ in each direction at the mesoscale.

\section{Experimental results}

\section{Results at the macroscopic scale.}

At the macroscopic scale, the displacement field is displayed in Fig. 4 for the horizontal component along direction $x_{1}$ that is perpendicular to the axis of the actuator (left figure) and for the vertical component along direction $x_{2}$ that is parallel to the axis of the actuator (right figure). The images are presented on a $10 \times 10$ - points grid. The components 11,22 , and 12 of the strain tensor are displayed in Figs. 5 and 6 It should be noted that the strain field is not homogeneous at the macroscopic scale that is due to the boundary conditions effects. In addition, in Fig. 4, it can be observed a slight loss of symmetry with respect to the median vertical line of the image. The loss of symmetry is explained by a misaligned loading. Consequently, the horizontal component of the resulting surface-force field applied by the actuator is non zero. It induces a shear strain but also a small horizontal slip of the specimen.
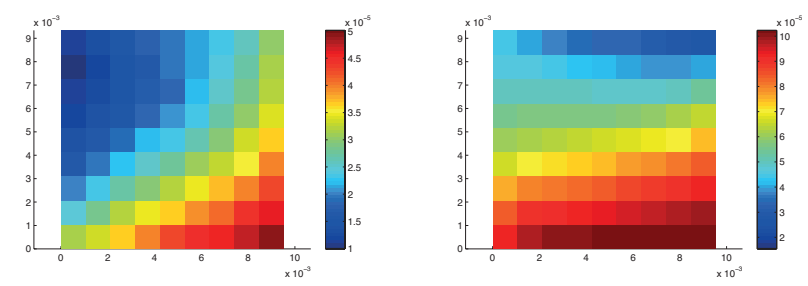

Figure 4: The horizontal axis is $x_{1}$ and the vertical axis is $x_{2}$. Component along direction $x_{1}$ of the displacement field (left figure) and along direction $x_{2}$ (right figure), at the macroscopic scale.

\section{Results at the mesoscopic scale.}

At the mesoscopic scale, the two components of the displacement field are displayed in Fig. 7 for direction $x_{1}$ (left figure) 

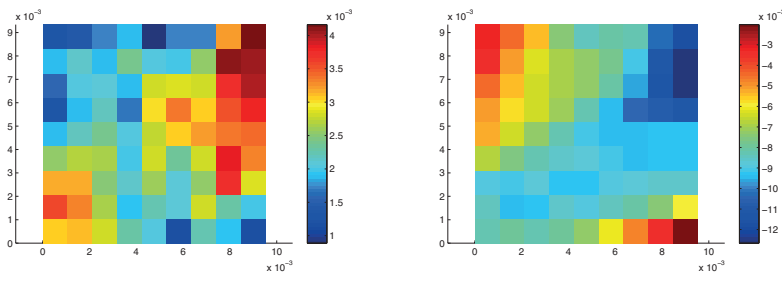

Figure 5: The horizontal axis is $x_{1}$ and the vertical axis is $x_{2}$. Component 11 (left figure) and component 22 (right figure) of the strain tensor at the macroscopic scale.

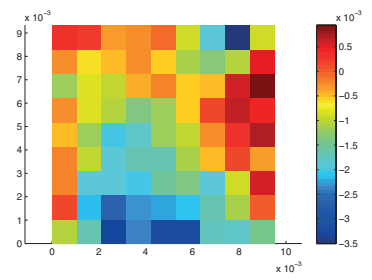

Figure 6: The horizontal axis is $x_{1}$ and the vertical axis is $x_{2}$. Component 12 (shear) of the strain tensor at the macroscopic scale.

and for direction $x_{2}$ (right figure). The images are presented with a $100 \times 100$ - points grid. The components 11,22 , and 12 of the strain tensor are displayed in Figs. 8 and 9 . Similarly to the macroscopic scale, a slight loss of symmetry is observed, which can be explained with the same reasons. Furthermore, in Fig 7, despite the vertical components of the displacement field is continue, an important gradient can be observed, which is not due to a crack.
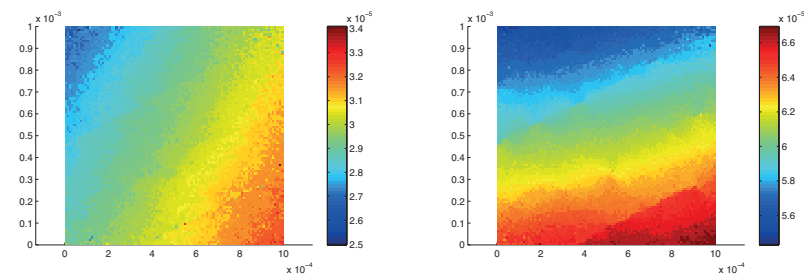

Figure 7: The horizontal axis is $x_{1}$ and the vertical axis is $x_{2}$. Component along direction $x_{1}$ of the displacement field (left figure) and along direction $x_{2}$ (right figure), at the mesoscopic scale (for image 42).
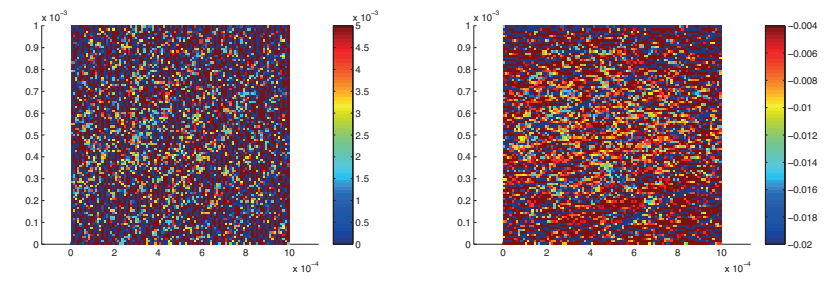

Figure 8: The horizontal axis is $x_{1}$ and the vertical axis is $x_{2}$. Component 11 (left figure) and component 22 (right figure) of the strain tensor at the mesoscopic scale(for image 42).

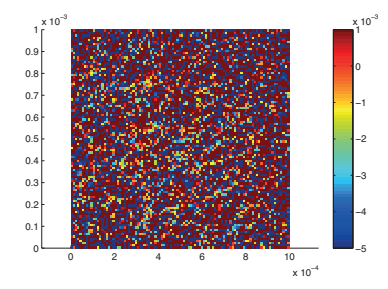

Figure 9: The horizontal axis is $x_{1}$ and the vertical axis is $x_{2}$. Component 12 (shear) of the strain tensor at the mesoscopic scale(for image 42).

\section{Brief summary of the statistical inverse problem}

The identification procedure proposed is based on the fact that the random apparent elasticity field at mesoscale of the microstructure cannot completely be identified by using only the measured displacement field at mesoscale. An additional information at macroscale is required. This is the reason why the identification at macroscale is introduced and consequently, two scales are simultaneously measured and used in the statistical inverse problem.

In this section, the identification procedure for solving the statistical inverse problem is briefly presented. Figure 10 presents an overview of the methodology that is detailed in Nguven (2013); Nguyen et al. (2015). The experimental displacement field $\mathbf{u}_{\text {exp }}^{\text {macro }}$ is constructed using a finite element interpolation of the experimental values of the displacement obtained on the $10 \times 10$ points correlation grid over the macroscopic domain. The strain field associated with $\mathbf{u}_{\mathrm{exp}}^{\text {macro }}$ is denoted by $\varepsilon_{\exp }^{\text {macro }}$. At macroscale, a deterministic boundary value problem is introduced for a three-dimensional linear elastic medium, which models the specimen in its experimental configuration (geometry, surface forces and Dirichlet conditions). The constitutive equation involves a prior deterministic model for the fourth-order elasticity tensor $C^{\text {macro }}(\mathbf{a})$ that is parameterized by a vector a. The tensor-valued strain field associated with this boundary value problem is denoted by $\varepsilon^{\text {macro }}(\mathbf{a})$ and also depends on a. Moreover, the experimental mesoscopic displacement field $\mathbf{u}_{\mathrm{exp}}^{\text {meso }}$ is constructed in using a finite element interpolation of the experimental values of the displacement obtained on the $100 \times 100$ points correlation grid over the mesoscopic domain. The strain field associated with $\mathbf{u}_{\mathrm{exp}}^{\text {meso }}$ is denoted by $\varepsilon_{\exp }^{\text {meso }}$. At mesoscale, a stochastic boundary value problem is introduced for a linear elastic medium in 2D plane stress with Dirichlet conditions defined by the values of the experimental displacements on the boundary of the mesoscopic correlation grid.

The apparent elasticity field is modeled as a prior tensorvalued random field and is considered as the restriction to subdomain $\Omega^{\text {meso }}$ of a statistically homogeneous random field $\left\{\mathbf{C}^{\text {meso }}(\mathbf{x} ; \mathbf{b}), \mathbf{x} \in \mathbb{R}^{3}\right\}$ in which $\mathbf{b}$ is the vector of the parameters. In using the Voigt notation, for all $\mathbf{x}$ fixed in $\Omega^{\text {meso }}$, the random fourth-order elasticity tensor $\mathbf{C}^{\text {meso }}(\mathbf{x})$ can be represented by a $(6 \times 6)$ real random matrix $\left[\mathbf{C}^{\text {meso }}(\mathbf{x})\right]$, which is the inverse matrix of a random compliance matrix 


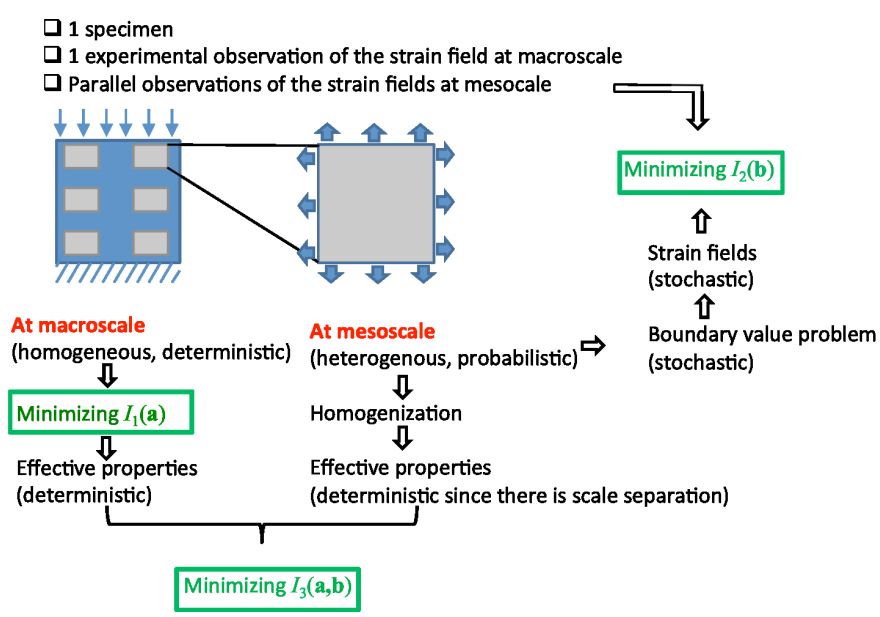

Figure 10: Overview of the methodology for the identification of a stochastic model of the apparent elasticity random field at mesoscopic scale.

$\left[\mathbf{S}^{\text {meso }}(\mathbf{x})\right]$. Nevertheless, we will keep the same notation for the strain fields and their matrix representations. For the case of $2 \mathrm{D}$ plane stresses, the prior stochastic model of $\left[\mathbf{C}^{\text {meso }}(\mathbf{x})\right]$ is constructed in choosing $\left[\mathbf{S}^{\text {meso }}\right]=\left\{\left[\mathbf{S}^{\text {meso }}(\mathbf{x})\right], \mathbf{x} \in \Omega^{\text {meso }}\right\}$ in the set $\mathrm{SFE}^{+}$(defined in Soize (2006, 2008, 2012)) of the non-Gaussian second-order stochastic fields with values in the set of all the positive-definite symmetric $(6 \times 6)$ real matrices. Hence, for a transverse anisotropic material in $2 \mathrm{D}$ plane stresses, we have $\mathbf{b}=\left(\delta, \ell, E_{T}, v_{T}\right)$ where $E_{T}$ and $v_{T}$ are the Young modulus and the Poisson coefficient that are independent of $\mathbf{x}$, and which parameterize the statistical mean value of the compliance matrix for the transverse isotropic material at the mesoscale (this statistical mean compliance matrix is independent of $\mathbf{x}$ because the elasticity random field is statistically homogeneous at the mesoscale). It should be noted that the identified values $E_{T}^{\text {meso }}$ and $v_{T}^{\text {meso }}$ of $E_{T}$ and $v_{T}$ do not represent statistical mean values of these Young modulus and Poisson coefficient because the mean compliance matrix is a nonlinear mapping of $E_{T}$ and $v_{T}$. The parameter $\delta$ of the stochastic model allows for controlling the level of the statistical fluctuations of the elasticity field at the mesoscale (for $\delta=0$ the statistical fluctuations are zero). The parameter $\ell$ is a length that characterizes the spatial correlation lengths of the elasticity random field at the mesoscale, which are assumed to be the same in the two directions.

In order to identify $\mathbf{a}$ and $\mathbf{b}$, three numerical indicators, $\mathcal{I}_{1}(\mathbf{a}), \mathcal{I}_{2}(\mathbf{b}), \mathcal{I}_{3}(\mathbf{a}, \mathbf{b})$, are introduced. The distance between $\varepsilon_{\exp }^{\text {macro }}$ and $\varepsilon^{\text {macro }}(\mathbf{a})$ is quantified by the first numerical indicator $\mathcal{I}_{1}(\mathbf{a})$. The numerical indicator $\mathcal{I}_{2}(\mathbf{b})$ quantifies the difference between the spatial fluctuations of the experimental strain field at mesoscale (around its volume average $\underline{\mathbb{E}}_{\exp }^{\text {meso }}$ ) and the statistical fluctuations of the random strain field of the stochastic boundary value problem (around its mean value $\mathbb{E}^{\text {meso }}$ ) at mesoscale. Finally, the numerical indicator $\mathcal{I}_{3}(\mathbf{a}, \mathbf{b})$ quantifies the distance between the effective elasticity matrix $\left[C^{\text {macro }}(\mathbf{a})\right]$ used in the deterministic boundary value problem at macroscale, and the effective elasticity matrix $\left[\mathbf{C}^{\text {eff }}(\mathbf{b})\right]$ calculated by homogenization of the mesoscale stochastic model in subdomain $\Omega^{\text {meso }}$ with the assumption that it is a REV. We then have

$$
\begin{gathered}
\mathcal{I}_{1}(\mathbf{a})=\left\|\varepsilon_{\exp }^{\text {macro }}-\varepsilon^{\text {macro }}(\mathbf{a})\right\| \|^{2}, \\
\mathcal{I}_{2}(\mathbf{b})=\int_{\Omega^{\text {meso }}}\left(\delta^{\text {meso }}(\mathbf{x} ; \mathbf{b})-\delta_{\exp }^{\text {meso }}\right)^{2} d \mathbf{x}, \\
\mathcal{I}_{3}(\mathbf{a}, \mathbf{b})=\left\|\left[C^{\text {macro }}(\mathbf{a})\right]-E\left\{\left[\mathbf{C}^{\mathrm{eff}}(\mathbf{b})\right]\right\}\right\|_{F}^{2} .
\end{gathered}
$$

in which $\|\cdot\|_{F}$ is the Frobenius norm of a matrix,

$$
\begin{gathered}
\|\| \varepsilon_{\exp }^{\text {macro }}-\varepsilon^{\text {macro }}(\mathbf{a})\|\|^{2}=\int_{\Omega^{\text {macro }}}\left\|\varepsilon_{\exp }^{\text {macro }}(\mathbf{x})-\varepsilon^{\text {macro }}(\mathbf{x} ; \mathbf{a})\right\|_{F}^{2} d \mathbf{x} . \\
\delta^{\text {meso }}(\mathbf{x} ; \mathbf{b})=\frac{\sqrt{V^{\text {meso }}(\mathbf{x} ; \mathbf{b})}}{\left\|\underline{\mathbb{E}}^{\text {meso }}(\mathbf{b})\right\|_{F}} \quad \text { and } \quad \delta_{\exp }^{\text {meso }}=\frac{\sqrt{V_{\exp }^{\text {meso }}}}{\left\|\underline{\mathbb{E}}_{\exp }^{\text {meso }}\right\|_{F}},
\end{gathered}
$$

and where

$$
\begin{gathered}
\underline{\mathbb{E}}^{\text {meso }}(\mathbf{b})=\frac{1}{\left|\Omega^{\text {meso }}\right|} \int_{\Omega^{\text {meso }}} \mathbb{E}^{\text {meso }}(\mathbf{x} ; \mathbf{b}) d \mathbf{x}, \\
\mathscr{\mathbb { E }}_{\exp }^{\text {meso }}=\frac{1}{\left|\Omega^{\text {meso }}\right|} \int_{\Omega^{\text {meso }}} \mathbb{E}_{\exp }^{\text {meso }}(\mathbf{x}) d \mathbf{x}, \\
V^{\text {meso }}(\mathbf{x} ; \mathbf{b})=E\left\{\left\|\mathbb{E}^{\text {meso }}(\mathbf{x} ; \mathbf{b})-\underline{\mathbb{E}}^{\text {meso }}(\mathbf{b})\right\|_{F}^{2}\right\}, \\
V_{\text {exp }}^{\text {meso }}=\frac{1}{\left|\Omega^{\text {meso }}\right|} \int_{\Omega^{\text {meso }}}\left\|\mathbb{E}_{\exp }^{\text {meso }}(\mathbf{x})-\underline{\mathbb{E}}_{\text {exp }}^{\text {meso }}\right\|_{F}^{2} d \mathbf{x}
\end{gathered}
$$

The identification of parameters $\mathbf{a}$ and $\mathbf{b}$ is obtained by solving a multi-objective optimization problem for the three indicators $\mathcal{I}_{1}(\mathbf{a}), \mathcal{I}_{2}(\mathbf{b})$, and $\mathcal{I}_{3}(\mathbf{a}, \mathbf{b})$. A genetic algorithm is used in order (1) to construct the Pareto front and (2) to find the global optimal values $\mathbf{a}^{\text {macro }}$ and $\mathbf{b}^{\text {meso }}$ of the multi-objective optimization problem.

The value of $\mathbf{a}^{\text {macro }}$ is almost unchanged through the iterations when the multi-objective problem is solved. Consequently, we found that the optimal value of $\mathbf{a}=\left(E_{T}^{\text {macro }}, v_{T}^{\text {macro }}\right)$ is $\mathbf{a}^{\text {macro }}=\left(6.74 \times 10^{9} \mathrm{~Pa}, 0.32\right)$. Less than 100 generations have been enough for solving the multi-objective optimization problem. Its optimal components are $\ell^{\text {meso }}=5.06 \times 10^{-5} \mathrm{~m}$, $\delta^{\text {meso }}=0.28, E_{T}^{\text {meso }}=6.96 \times 10^{9} \mathrm{~Pa}, v_{T}^{\text {meso }}=0.37$. This optimal solution yields a spatial correlation length equal to $5.06 \times 10^{-5} \mathrm{~m}$, which has to be compared to the spatial resolution that is $10^{-5} \mathrm{~m}$, and which is in agreement with the assumption introduced concerning the separation of the scales. This length is also of the same order of magnitude than the distance between adjacent lamellae or osteons in cortical bovine femur.

\section{Conclusion}

In the framework of a multiscale identification of the apparent elasticity field at the mesoscopic scale for a complex elastic microstructure, the measurements of the deformation of a specimen submitted to a single external loading must simultaneously be carried out for both the mesoscopic scale and the macroscopic scale. In this paper, an innovative 
experimental protocol has been presented for the multiscale measurements of a cortical bone specimen. A test bench, made up of a unique optical lens, has been developed, and uses a single imaging at the two scales. The specimen is a centimetric cube of cortical bone. Some difficulties have been induced by the nature of the cortical bone for which no existing speckled pattern technique was available for imaging the displacement field, simultaneously at the macroscopic scale and at the mesoscopic scale by the DIC method. The difficulties have been circumvented thanks to the development of an adapted experimental procedure. The presented experimental results are coherent and satisfying, and the use of these results have been used for a multiscale identification of a stochastic model of the apparent elasticity random field at mesoscopic scale.

\section{References}

Avril, S., Bonnet, M., Bretelle, A., Grédiac, M., Hild, F., Ienny, P., Latourte, F., Lemosse, D., Pagano, S., Pagnacco, E., \& Pierron, F. (2008a). Overview of identification methods of mechanical parameters based on full-field measurements. Experimental Mechanics, 48, 381-402.

Avril, S., \& Pierron, F. (2007). General framework for the identification of constitutive parameters from full-field measurements in linear elasticity. International Journal of Solids and Structures, 44, 4978-5002.

Avril, S., Pierron, F., Pannier, Y., \& Rotinat, R. (2008b). Stress reconstruction and constitutive parameter identification in plane-stress elastoplastic problems using surface measurements of deformation fields. Experimental Mechanics, (pp. 403-419).

Baxter, S. C., \& Graham, L. L. (2000). Characterization of random composites using a moving window technique. ASCE J Engng Mech, 126(4), 389-404.

Bertolino, G., Bilger, N., \& Crépin, J. (2007). Modeling microstructures and microstructural effects on macroscopic and intragranular mechanical behavior. Computational Materials Science, 40, 408-416.

Besnard, G., Hild, F., \& Roux, S. (2006). Finite-element displacement fields analysis from digital images: Application to portevin-le chatelier bands. Experimental Mechanics, 46, 789-803.

Bonnet, M., \& Constantinescu, A. (2005). Inverse problems in elasticity. Inverse Problems, 21, R1-R50.

Bornert, M., Brémand, F., Doumalin, P., Dupré, J., Fazzini, M., Grédiac, M., Hild, F., Mistou, S., Molimard, J., Orteu, J., Robert, L., Surrel, Y., Vacher, P., \& Wattrisse, B. (2009). Assessment of digital image correlation measurement errors: Methodology and results. Experimental Mechanics, 49, 353-370.

Bornert, M., Vales, F., Gharbi, H., \& Nguyen Minh, D. (2010). Multiscale full-field strain measurements for micromechanical investigations of the hydromechanical behaviour of clayey rocks. Strain, 46, 33-46.

Calloch, S., Dureisseix, D., \& Hild, F. (2002). Identification de modèles de comportement de matériaux solides: utilisation d'essais et de calculs. Technologies et Formations, 100, 36-41.

Chauvet, D., Carpentier, A., \& Allain, J.-M. (2010). Histological and biomechanical study of dura mater applied to the technique of dura splitting decompression in chiari type i malformation. Neurosurgical Review, 33, 287294.

Chevalier, L., Calloch, S., Hild, F., \& Marco, Y. (2001). Digital image correlation used to analyze the multiaxial behavior of rubber-like materials. European Journal of Mechanics A-Solids, 20, 169-187.

Constantinescu, A. (1995). On the identification of elastic moduli from displacement-force boundary measurements. Inverse Problems in Engineering, 1, 293-315.

Geymonat, G., Hild, F., \& Pagano, S. (2002). Identification of elastic parameters by displacement field measurement. Comptes Rendus Mécanique, 330, 403-408.

Geymonat, G., \& Pagano, S. (2003). Identification of mechanical properties by displacement field measurement: a variational approach. Meccanica, 38, 535-545.
Graham, L. L., Gurley, K., \& Masters, F. (2003). Non-gaussian simulation of local material properties based on a moving-window technique. Probabilistic Engineering Mechanics, 18, 223-234.

Gusachenko, I., Tran, V., Houssen, Y.-G., \& Allain, J.-M. (2012). Polarizationresolved second-harmonic generation in tendon upon mechanical stretching. Biophysical Journal, 102, 2220-2229.

Heripre, E., Dexet, M., Crépin, J., Gélébart, L., Roos, A., Bornert, M., \& Caldemaison, D. (2007). Coupling between experimental measurements and polycrystal finite element calculations for micromechanical study of metallic materials. International Journal of Plasticity, 23, 1512-1539.

Hild, F. (2002). CORRELI ${ }^{L M T}$ : a software for displacement field measurements by digital image correlation. LMT-Cachan, internal report 254 , .

Hild, F., Périé, J.-N., \& Coret, M. (1999). Mesure de champs de déplacements 2D par intercorrélation d'images: CORRELI 2D. LMT-Cachan, internal report 230 ,

Hild, F., Raka, B., Baudequin, M., Roux, S., \& Cantelaube, F. (2002). Multiscale displacement field measurements of compressed mineral-wool samples by digital image correlation. Applied Optics, 41, 6815-6828.

Hild, F., \& Roux, S. (2006). Digital image correlation: from displacement measurement to identification of elastic properties - a review. Strain, 42, 69-80.

Hild, F., \& Roux, S. (2012). Comparison of local and global approaches to digital image correlation. Experimental Mechanics, 52, 1503-1519.

Houssen, Y.-G., Gusachenko, I., Schanne-Klein, M.-C., \& Allain, J.-M. (2011). Monitoring micrometer-scale collagen organization in rat-tail tendon upon mechanical strain using second harmonic microscopy. Journal of Biomechanics, 44, 2047-2052.

Jeulin, D. (1987). Microstructure modeling by random textures. Journal de Microscopie et de Spectroscopie Electroniques, 12, 133140.

Jeulin, D. (1989). Morphological modeling of images by sequential random functions. Signal Processing, 16, 403431.

Jeulin, D. (2001). Caractérisation morphologique et modèles de structures aléatoires. In Homogénéisation en mécanique des matériaux 1. Hermès Science Publications, Paris.

Kahnjetter, Z. L., Jha, N. K., \& Bhatia, H. (1994). Optimal image correlation in experimental mechanics. Optical Engineering, 33, 1099-1105.

Kalouche, I., Crépin, J., Abdelmoumen, S., Mitton, D., Guillot, G., \& Gagey, O. (2010). Mechanical properties of glenoid cancellous bone. Clinical Biomechanics, 25, 292298.

Madi, K., Forest, S., Boussuge, M., Gailliegue, S., Lataste, E., Buffiere, J.Y., Bernard, D., \& Jeulin, D. (2007). Finite element simulations of the deformation of fused-cast refractories based on X-ray computed tomography. Computational Materials Science, 39, 224-229.

Nguyen, M. (2013). Identification multi-échelle du champ d'élasticité apparent stochastique de microstructures hétérogènes - Application un tissu biologique. Thèse de doctorat de l'Université Paris-Est, Paris.

Nguyen, M., C., D., C., S., J.M., A., \& H., G. (2015). Multiscale identification of the random elasticity field at mesoscale of a heterogeneous microstructure using multiscale experimental observations. International Journal for Multiscale Computational Engineering, 13(4), 281-295.

Pan, B., Qian, K. M., Xie, H. M., \& Asundi, A. (2009). Two-dimensional digital image correlation for in-plane displacement and strain measurement: a review. Measurement Science and Technology, 20, 062001.

Pan, B., Xie, H. M., Xu, B. Q., \& Dai, F. L. (2006). Performance of sub-pixel registration algorithms in digital image correlation. Measurement Science and Technology, 17, 1615-1621.

Rasband, W. (2013). Imagej. U. S. National Institutes of Health, Bethesda, Maryland, USA, http://imagej.nih.gov/ij, 1997-2014.

Rethore, J., Tinnes, J.-P., Roux, S., Buffiere, J.-Y., \& Hild, F. (2008). Extended three-dimensional digital image correlation (X3D-DIC). Comptes Rendus Mécanique, 336, 643-649.

Roux, S., \& Hild, F. (2008). Digital image mechanical identification (DIMI). Experimental Mechanics, 48, 495-508.

Roux, S., Hild, F., \& Berthaud, Y. (2002). Correlation image velocimetry: a spectral approach. Applied Optics, 41, 108-115.

Roux, S., Hild, F., Viot, P., \& Bernard, D. (2008). Three-dimensional image correlation from X-ray computed tomography of solid foam. Composites Part A-Applied Science and Manufacturing, 39, 1253-1265.

Schreier, H. W., Braasch, J. R., \& Sutton, M. A. (2000). Systematic errors in digital image correlation caused by intensity interpolation. Optical Engineering, 39, 2915-2921. 
Soize, C. (2006). Non-gaussian positive-definite matrix-valued random fields for elliptic stochastic partial differential operators. Computer Methods in Applied Mechanics and Engineering, 195, 26-64.

Soize, C. (2008). Tensor-valued random fields for meso-scale stochastic model of anisotropic elastic microstructure and probabilistic analysis of representative volume element size. Probabilistic Engineering Mechanics, 23, 307323.

Soize, C. (2012). Stochastic Models of Uncertainties in Computational mechanics volume 2 of Lecture Notes in Mechanics. Reston: American Society of Civil Engineers.

Sutton, M. A., Yan, J. H., Tiwari, V., Schreier, H. W., \& Orteu, J. J. (2008). The effect of out-of-plane motion on $2 \mathrm{~d}$ and $3 \mathrm{~d}$ digital image correlation measurements. Optics and Lasers in Engineering, 46, 746-757.

Vendroux, G., \& Knauss, W. G. (1998a). Submicron deformation field measurements: Part 1. developing a digital scanning tunnelling microscope. Experimental Mechanics, 38, 18-23.

Vendroux, G., \& Knauss, W. G. (1998b). Submicron deformation field measurements: Part 2. improved digital image correlation. Experimental Mechanics, 38, 86-92.

Yamaguchi, H., Kikugawa, H., Asaka, T., Kasuya, H., \& Kuninori, M. (2011). Measurement of cortical bone strain distribution by Image Correlation Techniques and from fracture toughness. Materials Transactions, 52(5), 10261032.

Yang, D. S., Bornert, M., Chanchole, S., Gharbi, H., Valli, P., \& Gatmiri, B. (2012). Dependence of elastic properties of argillaceous rocks on moisture content investigated with optical full-field strain measurement techniques. International Journal of Rock Mechanics and Mining Sciences, 53, 45-55.

Yang, D. S., Bornert, M., Gharbi, H., Valli, P., \& Wang, L. (2010). Optimized optical setup for dic in rock mechanics. 14th International Conference on Experimental Mechanics, (pp. 22019-1-8). 